International Journal of Engineering \& Technology, $7(3.36)(2018) 52-55$
SPC
Website: $w w w . s c i e n c e p u b c o . c o m / i n d e x . p h p / I J E T$
Research paper

\title{
Overview on the Form-Finding of Tensegrity Structure
}

\author{
A.H. Nadia Farhana ${ }^{1}$, C. L. OH ${ }^{1 *}$, H. M. Yee ${ }^{2}$, A. Anizahyati ${ }^{1}$ \\ ${ }^{1}$ Institute for Infrastructure Engineering and Sustainable Management, \\ Faculty of Civil Engineering, Universiti Teknologi MARA \\ 40450 Shah Alam, Selangor, Malaysia \\ ${ }^{2}$ Faculty of Civil Engineering, Universiti Teknologi MARA \\ Cawangan Pulau Pinang, Kampus Permatang Pauh, Jalan Permatang Pauh \\ 13500 Permatang Pauh | Pulau Pinang \\ *Corresponding author E-mail: chailian@salam.uitm.edu.my
}

\begin{abstract}
This paper presents an overview of the previous research on the form finding structures which include tensegrity and biotensegrity structure. Tensegrity systems have been widely used for many applications due to the stability and flexibility of the structures. On technologies era nowadays, a stable structure is needed to implement for new developments especially robotics and machineries. Therefore, the importance of finding the shape or design configuration is to ensure it is in self-equilibrium which able to support itself. In order to build new structures which lies on general requirement, previous researchers have proposed several form-finding methods for instance by using force density method, advance force density method, finite element method, dynamic relaxation method, genetic algorithm, novel linear approach and Monte Carlo method. What kind of stable structures can be produced and how efficient the structures by using those methods? In this paper, understanding on the form-finding methods and various numerical examples of form-finding conducted in previous studies are presented to enhance researchers' knowledge on the form-finding of tensegrity structures.
\end{abstract}

Keywords: tensegrity, biotensegrity, form-finding, geometry, equilibrium.

\section{Introduction}

Buckminster Fuller has coined the term tensegrity to name a spatial structure such as X-Piece built by Kenneth Snelson in 1948 [1]. Tensegrity structure consists of a set of compression elements (discontinuous struts) and tensional elements (continuous cable) which formed in self-equilibrium condition. Tensegrity structures have been suggested for many applications such as medication engineering, treatment strategies, automation and robotics. The tensegrity structures mimicking biology system or biotensegrity has also been proposed. Owing to the needs to find stable and selfequilibrium configurations, exploration on efficient form-finding methods for tensegrity structures are very essential.

Form-finding can be defined as finding the stable shape or configuration of a structure which satisfies static equilibrium condition [2]. The determination of a stable and self-equilibrium form is the key process in designing many structures such as tensegrity and biotensegrity. Therefore, several steps in determination of nodal coordinates, establishment of system of equilibrium equations, satisfaction of self-equilibrium condition and solution for the axial forces are needed. Many form-findings methods employing different approaches have been proposed [3]. In this paper, overview of the research works specifically on the form-finding of tensegrity structure is presented. Through this paper, overviews of formfinding methods for tensegrity structures as well as explanation of some numerical examples may enhance readers knowledge on this topic.

The remaining paper is organized as follows. The form-finding methods used to determine the self-equilibrium configuration of tensegrity models and numerical examples of form-finding are discussed in Section 2 and Section 3, respectively. Lastly, the concluding remarks are addressed in Section 4.

\section{Form-Finding Methods}

Tibert and Pellegrino has classified the form-finding methods into kinematical and statical methods [3]. This section discussed the form-finding methods for tensegrity structures based on these two categories. Both categories aims in finding the configuration of the structure in self-stress condition.

\subsection{Kinematical Methods}

Kinematical methods determine the geometry of a structure involving maximization of strut lengths with unchangeable of cable length or minimization of cable lengths with unchangeable of strut length [3]. These methods do not acquire the cables in a pretension condition similarly as general method to build tensegrity structures. Typical kinematical methods are dynamic relaxation method, finite element method and Monte Carlo method. In this section, investigation works on tensegrity structures using these methods are explained.

Dynamic relaxation method is applicable for small scale projects in finding new shapes of a structure which was introduced by Motro [4]. This method employs nonlinear equilibrium equations with damping (i.e. irregular motion) in form-finding of tensegrity structures [5]. Iteration steps by mimicking a pseudo dynamic in time process which involve repetitive formulation of velocities and nodal forces begins with an initial configuration until satisfaction of equilibrium condition [6]. Dynamic relaxation method can 
only be applied to the known configuration and identified coordinates with the consideration of the equilibrium state and external forces of the structures [4]. This method has been used by Motro [4], Zhang and Ohsaki [7] as a form-finding method for irregular shapes of tensegrity structures. However, the finding is not very effective for large scale project [3].

Next, Monte Carlo method has been introduced by Metropolis and Ulam to determine new shape for tensegrity structure [8]. The operation of the form-finding method is easy and it can be used for large scale projects in finding either regular or irregular shapes of the tensegrity structures [6]. The method mimics a stochastic process that involving probability distribution function in solving form-finding problems [6]. This method determines a stable structure which is in equilibrium condition through trial configurations that randomly generated over time, followed by an acceptancerejection algorithm.

Finite element method is also suitable for determining configuration of a self-equilibrated tensegrity structure. This method presented by Pagitz and Mirats Tur [9] ensures the elements of tensegrity structures (i.e. cables and struts) are in equilibrium condition and with no axial stiffness. They have also stated that finite element method considers symmetry space of the configuration and uses an iteration to demonstrate the best configuration [9]. So, the stable configuration may be produced by applying this method even though it probably takes more time to get the form of the structure.

Other than that, Holland [10] and Paul et al. [11] proposed genetic algorithms to develop an initial configuration into a stable structure for any types of shapes [12]. It has been also used by Paul et al. [11], Xu and Luo [13] as a part of their form-finding methods which promises to build a super-stable structure [14]. Genetic algorithm is used to minimize the problem of the function that involves in eigenvalues of the force density matrix [14]. This method leads a set of force densities to get required rank deficiency using simpler computation [14] with less design variables of tensegrity structures [15].

\subsection{Statical Methods}

Statical methods determines a stable configuration for tensegrity structure which involves both topology of the structure (i.e. nodes and elements) as well as forces acting on the structures [3]. Common statical methods are force density method, advanced density method and novel linear approach.

Force density method has been proposed by Linkwitz and Schek [16] to determine the nodal coordinates of the cable and struts by considering forces and geometry (i.e. length) of the tensegrity structure. Force densities variables of pre-specified topology are resolved by using linear equation to achieve required rank deficiency [16]. This method is suitable to obtain new configurations especially for symmetry and regular tensegrity structures.

Zhang and Ohsaki introduced adaptive force density method because the initial force density method is not very suitable for structures with many members and irregular shape [17]. The proposed method is a continuation from the initial force density method formulation and adapt eigenvalue analysis to find a feasible set of force densities based on required rank deficiency [17]. Then, the self-equilibrated configurations of the structure can be determined by specifying a set of independent nodal coordinates.

Moreover, Energy Optimization method is proposed by Li, Skelton and Yan to design the configuration of deployable tensegrity structure with minimal energy [18]. This method used to construct a structure that can deploy itself to form any shapes by restoring the energy in tensional element without applying external forces. When optimizing the energy, the initial constrain force need to be as small as possible to make sure the problem becomes a constrained optimization problem [18]. Then, the model can be constructed by solving the force density of each cable and the equilibrium condition of the structure can be determined to form the stable structure.
Moghaddas and Choong [19] employed novel linear approach. They introduced this method due to unsolved linear equation and unsymmetrical condition for the form finding of prism tensegrity structure. This method is not focusing on solving the equilibrium equations because the number of equilibrium equations for a prism tensegrity system is equal to the number of its joints. The structures need to be in symmetrical condition to solve the linear equations and not required other conditions or iteration process. By using other methods with nonlinear calculations, it is applicable for regular structures with fewer members [19]. However, these methods are able to create regular and irregular prism tensegrity configuration accurately with numerous members.

There are two categories of methods reviewed in this paper which is kinematical and statical method. Considering both methods, it is concluded that kinematical methods are suitable to determine the configuration based on maximization and minimization of the strut or cable lengths while statical methods are suitable to search for equilibrium configurations in pre-stress condition. All submethods presented have their advantages and disadvantages due to the suitability of form of structures, thus, only Monte Carlo methods and adaptive of force density method are available for any shape of regular and irregular structures.

\section{Numerical Examples of Form-Finding}

Every structure has been modelled by various design or shape. By using the form-finding method explained on previous section, the configuration of new structure can be designed easily in a stable condition. Therefore, there are many numerical examples of formfinding that has been developed by previous researcher which can be categorized as regular design, irregular design and biotensegrity design.

\subsection{Regular Design}

This section presents numerical example of form-finding for regular tensegrity such as hexagonal, prisms, octahedron, icosahedron, tetrahedron, cylindrical, tower and arch tesnegrity. Tensegrity is considered as regular design when the structure is in symmetrical shapes which have equal sides and angles. Most of researchers have studied on this type of design because the equilibrium condition of the configuration could be found easily compared to irregular one. Figure 1 shows symmetry configurations are regular and satisfied the nodal equilibrium [3].

Hexagonal tensegrity (Figure 1(a)), prism tensegrity (Figure 1(b)) and octahedron tensegrity (Figure 1(c)) have been commonly investigated by using force density method because this method uses simple mathematical solution [3]. Prism tensegrity has also been studied by using novel linear approach that does not employ any iteration processes as well as genetic algorithm in the determination of equilibrium condition by solving a defined optimisation problem. On the one hand, octahedron tensegrity has also been studied by Monte Carlo method, genetic algorithm and adaptive force density method.

Other than that, icosahedron tensegrity and tetrahedron tensegrity have been studied by using genetic algorithm, force density method, Monte Carlo method and analytical solution. Both tensegrities have a solid shape with complex geometry and topology. These form-finding methods have similarity in the assumptions in the form-finding analysis and the comparison of the form-finding results are in good agreement [6]. Figure 1(d) and Figure 1(e) shows the final configurations searched by using genetic algorithm and force density method, respectively. 


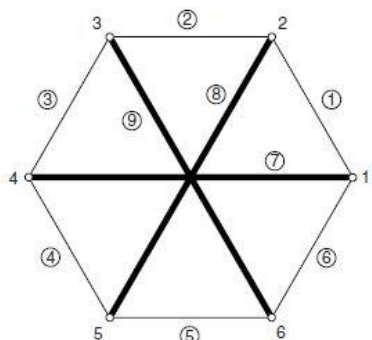

(a)

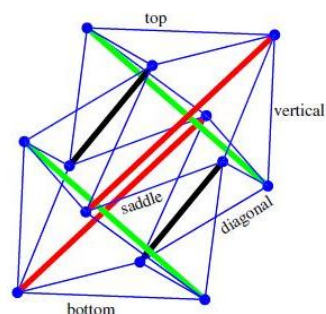

(c)

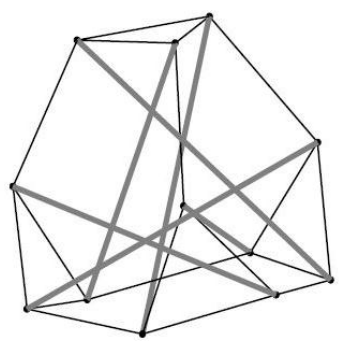

(e)

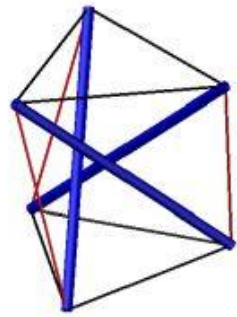

(b)

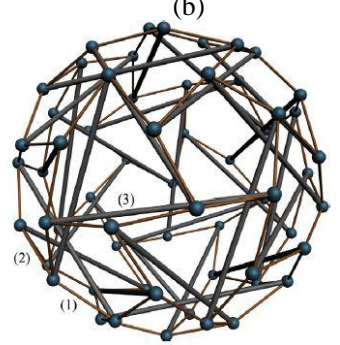

(d)

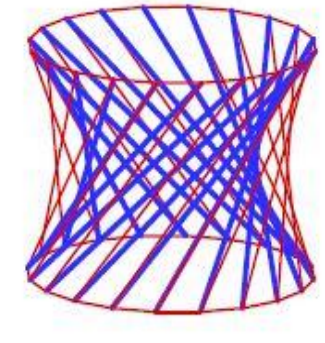

(f)
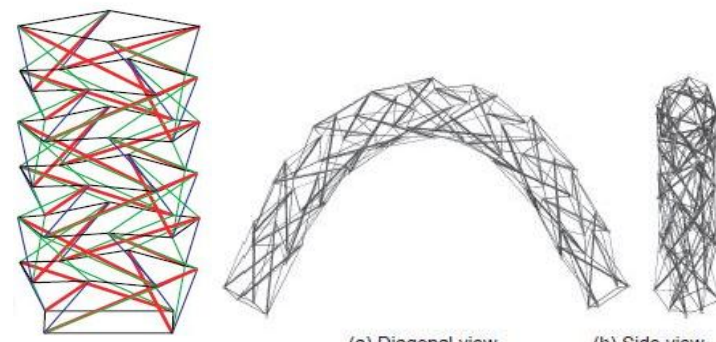

(a) Diagonal view

(b) Side view
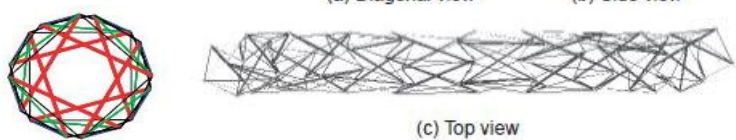

(c) Top view

(g)

Fig. 1: Numerical examples of regular design produced using several methods (a) hexagonal tensegrity [3], (b) prism tensegrity [19], (c) octahedron tensegrity [17], (d) icosahedron tensegrity [24], (e) tetrahedron tensegrity [20], (f) cylindrical tensegrity [6], (g) tower-like tensegrity [7], (h) arch-like tensegrity [7].

Moreover, cylindrical tensegrity structures have been studied by using Monte Carlo form-finding, dynamic relaxation and force density method. The methods can be used to determine the equilibrium condition of the cylindrical tensegrity configuration which also known as large scale structures [6]. Figure 1(f) shows an example of cylindrical tensegrity structure with positive stiffness and in self-stress condition [20].

Tower-like tensegrity structure consists of several stages of prismatic tensegrity structure with same size is shown in Figure $1(\mathrm{~g})$ [7]. Ten-stage tower-like tensegrity has been investigated by using dynamic relaxation method by Zhang and Ohsaki [7] whereas Masic et al. studied six-stage tower-like tensegrity by using algebraic approach [21] which adopted force density method in the form-finding process. Oh et al. suggest the tapered three-stage tensegrity model searched in the study for deployable structure [22].
Figure 1(h) shows an arch tensegrity structure successfully searched by using dynamic relaxation method. The initial configuration of this structure combines ten units prismatic tensegrity structure in order to get the final arch configuration [7]. The establishment of arch tensegrity process is same as tower-like tensegrity structure where several units of tensegrity structure are stacked up but with an arch-like.

\subsection{Irregular Design}

This section presents numerical example of form-finding for irregular tensegrity such as in Figure 2. Irregular design is the structures produced are asymmetrical shapes (not having equal sides and angles). There are not many researchers studied on this type of design because a more complex formulation involved in obtaining self-equilibrated configuration.

Several designs for irregular tensegrity structure have been developed from Monte Carlo form-finding method and novel linear approach as shown in Figure 2. For Monte Carlo method, irregular design has generally been determined by using regular shape as an initial configuration [6]. Complex prism tensegrity structure has also been explored by using novel linear approach is unsymmetrical and consists more number of members and joints [6].

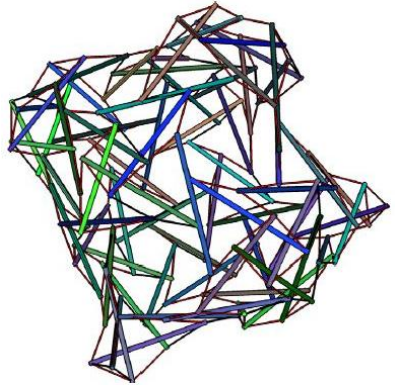

(a)

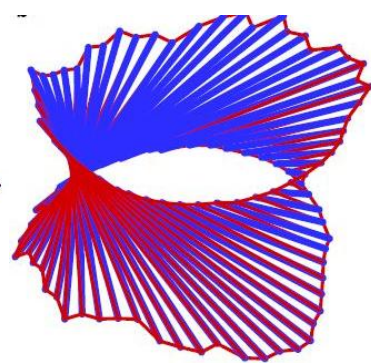

(b)

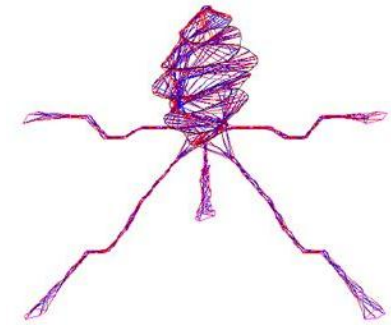

(c)

Fig. 2: Numerical examples of irregular design produced by using several methods (a) fully irregular tensegrity [6] (b) irregular cylindrical tensegrity [6] (c) complex multi-layer prism tensegrity [19].

\subsection{Biotensegrity Design}

Biotensegrity design means structures produced followed the human body geometries. Previous researchers have developed the biotensegrity model mimicking spine, foot, elbow and DNA. Figure 3 shows various model or configuration build based on human-made tensegrity to biology systems.

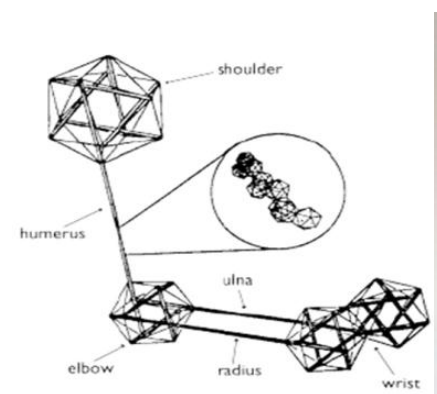

(a)

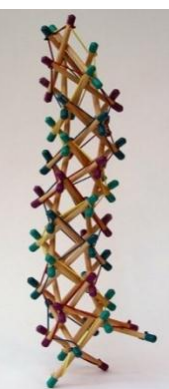

(b)

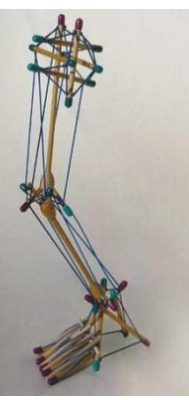

(c) 


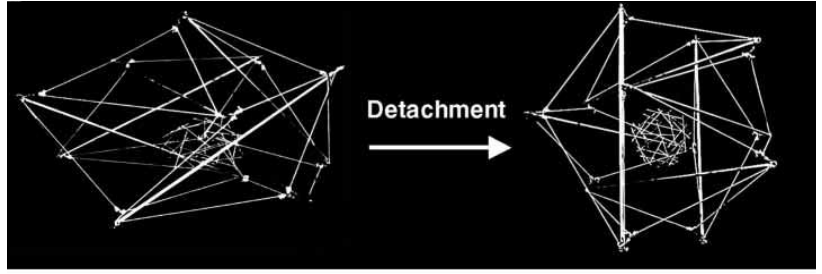

(d)

Fig. 3: Numerical examples of biotensegrity designs (a) elbow [24], (b) spine [25], (c) foot [25], (d) DNA [26].

Biotensegrity structure has been seen in many applications such as medication engineering, structural engineering and treatment strategies. By applying the system, the ability of human cell can be predicted which contributes to the movements made by human, the construction of spatial structure contributes to the aesthetic value of the structure and it helps athletes to deal with joints or muscles problem. Form-finding for biotensegrity structure has been conducted. For instance, form-finding strategies for spine biotensegrity structure can be found in Oh et al. [27].

\section{Conclusions}

Form-finding is an important step to determine self-equilibrated configurations of tensegrity or biotensegrity structures. This paper reviews form-finding methods that were classified under two categories (i.e. kinematical method and statical method). Many methods and parameters were taken into account to solve the geometrical and forces problems throughout the process of form-finding for stable configuration of tensegrity structures. Each method presented previously have different advantages but focusing on similar goals. However, not all methods are suitable for certain cases such as for irregular configuration or for structures with more members. On the other hand, this paper also reviews numerical examples of tensegrity particularly the regular design, irregular design and biotensegrity design that have been successfully searched by using several methods likes force density method, genetic algorithm, Monte Carlo method, novel linear approach, dynamic relaxation and adaptive force density method. To this end, there is always a need to continuously investigate and apply formfinding methods which are either a new one or the modified method to improve the process especially in form-finding new form of tensegrity structures.

\section{Acknowledgement}

The authors would like to thank Ministry of Higher Education Malaysia through FRGS grant (FRGS/1/2017/TK01/UITM/02/1) for funding this study. We would like to express our appreciation to University Teknologi MARA (UiTM), Selangor in providing the facilities.

\section{References}

[1] K. D. Snelson, "Continuous tension, discontinuous compression structures," 1965.

[2] W. J. Lewis, Tension structures: form and behaviour. Thomas Telford, 2003.

[3] A. G. Tibert and S. Pellegrino, "Review of Form-Finding Methods for Tensegrity Structures," Int. J. Sp. Struct., vol. 18, no. 4, pp. 209-223, 2003.

[4] R. Motro, "Tensegrity systems: the state of the art," Int. J. Sp. Struct., vol. 7, no. 2, pp. 75-83, 1992.

[5] A. Szcześniak and A. Stolarski, "Dynamic Relaxation Method with Critical Damping for Nonlinear Analysis of Reinforced Concrete Elements."
[6] Y. Li, X. Q. Feng, Y. P. Cao, and H. Gao, "A Monte Carlo formfinding method for large scale regular and irregular tensegrity structures," Int. J. Solids Struct., 2010.

[7] J. Zhang and M. Ohsaki, "Form-Finding of Complex Tengrity Structures By Dynamic Relaxation," vol. 81, no. 719, pp. 71-77, 2016.

[8] N. Metropolis and S. Ulam, "The Monte Carlo Method," J. Am. Stat. Assoc., vol. 44, no. 247, pp. 335-341, 1949.

[9] M. Pagitz and J. M. Mirats Tur, "Finite element based form-finding algorithm for tensegrity structures," Int. J. Solids Struct., vol. 46, no. 17, pp. 3235-3240, 2009.

[10] J. H. Holland, Adaptation in natural and artificial systems: an introductory analysis with applications to biology, control, and artificial intelligence. MIT press, 1992

[11] C. Paul, H. Lipson, and F. V. Cuevas, "Evolutionary development of tensegrity structures.," Biosystems., vol. 101, no. 3, pp. 167-76, 2010.

[12] S. Juan Hernandez and J. M. Mirats Tur, "IRI - Tensegrity frameworks: static analysis review," no. February 2009.

[13] X. Xian and Y. Luo, "Form-finding of nonregular tensegrities using a genetic algorithm," Mech. Res. Commun. - MECH RES COMMUN, vol. 37, pp. 85-91, 2010.

[14] K. Koohestani, "Form-finding of tensegrity structures via genetic algorithm," Int. J. Solids Struct., vol. 49, no. 5, pp. 739-747, 2012.

[15] M. Yamamoto, B. S. Gan, K. Fujita, and J. Kurokawa, "A genetic algorithm based form-finding for tensegrity structure," Procedia Eng., vol. 14, pp. 2949-2956, 2011.

[16] K. Linkwitz and H. J. Schek, "Einige Bemerkungen zur Berechnung von vorgespannten Seilnetzkonstruktionen," Arch. Appl. Mech., vol. 40, pp. 145-158, 1971.

[17] J. Y. Zhang and M. Ohsaki, "Adaptive force density method for form-finding problem of tensegrity structures," Int. J. Solids Struct., vol. 43 , no. $18-19$, pp. 5658-5673, 2006.

[18] Q. Li, R. E. Skelton, and J. Yan, "Energy optimization of deployable tensegrity structure," Proc. 30th Chinese Control Conf., no. 3, pp. 2146-2151, 2011.

[19] M. Moghaddas and K. K. Choong, "A novel practical approach for the form finding of the prism tensegrity," pp. 1360-1370, 2016

[20] G. G. Estrada, H. J. Bungartz, and C. Mohrdieck, "Numerical formfinding of tensegrity structures," Int. J. Solids Struct., vol. 43, no. $22-23$, pp. 6855-6868, 2006.

[21] M. Masic, R. E. Skelton, and P. E. Gill, "Algebraic tensegrity formfinding,” Int. J. Solids Struct., vol. 42, no. 16-17, pp. 4833-4858, 2005.

[22] C.L. Oh, K.K. Choong, T. Nishimura, S.W. Lee, "Self-equilibrated Tapered Three-stage Tensegrity Mast," Journal of Physics: Conference Series, vol. 1005, no. 1, p. 012039, 2018.

[23] K. Koohestani and S. D. Guest, "A new approach to the analytical and numerical form-finding of tensegrity structures," Int. J. Solids Struct., vol. 50, no. 19, pp. 2995-3007, 2013.

[24] S. M. Levin, "Putting the shoulder to the wheel: A new biomechanical model for the shoulder girdle," Biomed. Sci. Instrum., vol. 33, no. February 1997, pp. 412-417, 1997.

[25] T. Flemons, "Biotensegrity Model," Intension Designs. [Online]. Available: http://intensiondesigns.ca/models/. [Accessed: 30-Aug2018].

[26] D. E. Ingber, "Tensegrity I. Cell structure and hierarchical systems biology," J. Cell Sci., vol. 116, no. 7, pp. 1157-1173, 2003.

[27] C.L. Oh, K.K. Choong, T. Nishimura, J.Y, Kim, "Form-finding of Human Spine Inspired Biotensegrity," Proceedings of IASS Annual Symposia, vol. 2016, no. 21, pp. 1-10, 2016. 